\title{
Right cerebral hemisphere and central auditory processing in children with developmental dyslexia
}

\author{
El hemisferio cerebral derecho y el procesamiento auditivo central en niños \\ con dislexia del desarrollo
}

Paulina C. Murphy-Ruiz', Yolanda R. Peñaloza-López², Felipe García-Pedroza³, Adrián Poblano4

\begin{abstract}
Objective: We hypothesized that if the right hemisphere auditory processing abilities can be altered in children with developmental dyslexia (DD), we can detect dysfunction using specific tests. Method: We performed an analytical comparative cross-sectional study. We studied 20 right-handed children with DD and 20 healthy right-handed control subjects (CS). Children in both groups were age, gender, and school-grade matched. Focusing on the right hemisphere's contribution, we utilized tests to measure alterations in central auditory processing (CAP), such as determination of frequency patterns; sound duration; music pitch recognition; and identification of environmental sounds. We compared results among the two groups. Results: Children with DD showed lower performance than CS in all CAP subtests, including those that preferentially engaged the cerebral right hemisphere. Conclusion: Our data suggests a significant contribution of the right hemisphere in alterations of CAP in children with DD. Thus, right hemisphere CAP must be considered for examination and rehabilitation of children with DD.
\end{abstract}

Keywords: dyslexia, right hemisphere, left hemisphere, cerebral lateralization, brain asymmetry, central auditory processing disorders.

\section{RESUMEN}

Objectivo: Examinamos si las habilidades del procesamiento auditivo central (PAC) del hemisferio derecho se encuentran alteradas en niños con dislexia del desarrollo (DD), por medio de pruebas específicas. Método: Realizamos un estudio comparativo transversal. Estudiamos 20 niños diestros con DD y 20 niños controles (C) sanos. Los niños de ambos grupos fueron pareados por edad, género y grado escolar. Utilizamos varias pruebas para medir alteraciones del PAC, tales como: determinación de patrones de frecuencia; duración del sonido; reconocimiento de tono musical e identificación de sonidos ambientales, enfatizando la contribución del hemisferio derecho. Comparamos los resultados entre los niños con DD y C. Resultados: Los niños con DD mostraron menores puntuaciones que los C en todas las subpruebas del PAC, incluyendo aquellas que involucran preferencialmente al hemisferio cerebral derecho. Conclusión: Los datos obtenidos sugieren una contribución significativa del hemisferio derecho para producir alteraciones del PAC en niños con DD. Por lo tanto, las PAC que involucran al hemisferio derecho deben ser consideradas en la evaluación y rehabilitación de niño s con DD.

Palabras clave: dislexia, hemisferio derecho, hemisferio izquierdo, lateralización cerebral, asimetría cerebral, procesamiento auditivo central.

The brain decodes information in an integrated double hemisphere $\operatorname{process}^{1}$. In this functional organization, each cerebral hemisphere is specialized in some functions and plays a critical and complementary role where interhemispheric communication is very important ${ }^{2}$. The left hemisphere is specialized in verbal-auditory analysis, while the right hemisphere decodes non-verbal stimuli. Language functions related to right hemisphere processing are primarily pragmatics; analysis of prosodic information; recognition of voices, natural sounds, melodies; and the processing of simple and complex auditory patterns ${ }^{3}$.

Music is another complex auditory stimuli. For its perception, both hemispheres are required; the left hemisphere is specialized in the extraction of temporal sound features

\footnotetext{
${ }^{1} \mathrm{MD}$, Audiologist. Department of Audiology and Communication Disorders, National Institute of Rehabilitation, Mexico City, México;

${ }^{2}$ MD, M.Sc, Audiologist. Laboratory of Central Auditory Processes Alterations Research. National Institute of Rehabilitation. Mexico City, México;

${ }^{3}$ MD, M.P.H., Department of Familial Medicine. School of Medicine (UNAM). Mexico City, México;

${ }_{4}^{4}$ MD, DSc, Neurophysiologist. Laboratory of Cognitive Neurophysiology, National Institute of Rehabilitation, Mexico City, México.

Correspondence: Adrián Poblano; Av. México-Xochimilco 289; Colonia Arenal de Guadalupe; Deleg. Tlalpan; cp 14389 Mexico City - México;

E-mail:drdyslexia@latinmail.com

Conflicts of interest: There is no conflicts of interest to declare.

Received 12 February 2013; Received in final form 11 June 2013; Accepted 18 June 2013.
} 
while the right hemisphere decodes primarily the spectra of frequencies 4 .

For reading, the right hemisphere enables the correct use of spaces, identification of the left part of the text, orthography, oral reading melody, emotional content of words and non-words, and some lexical responses ${ }^{5}$. All these activities are critical functions for the acquisition of reading and writing.

Developmental dyslexia (DD) is an alteration of learning to read and is manifested by severe difficulties in reading and writing, often after a maturational language delay, resulting in an alteration of the establishment of temporospatial analysis relationships needed for these functions ${ }^{6}$. DD affects 5\%-10\% of the child population ${ }^{7}$, and some studies reported $17 \%$ of prevalence. In subjects affected by DD, different associated alterations of central auditory processing (CAP) have been reported previously, but few researcher have studied the contribution of cerebral right hemisphere dysfunction to these alterations ${ }^{8,9}$. The main right hemisphere-related alterations in writing and reading disorders were characters, syllables, and number production alterations; inversion or rotations of graphemes; grapheme confusions (primarily those with identical appearances); and writing in mirror image among others symptoms ${ }^{10}$. DD seems to have a complex genetic and environmental basis ${ }^{11}$. Pathophysiology is not well known in its whole picture, and several theories have been proposed, such as the phonologic decoding alteration, fast and not appropriated auditory processing, visual perception dysfunction, cerebellar damage, and the injury to the visual magnocellular subsystem ${ }^{12-14}$. Several cortical and subcortical malformations, and neural migration disorders have been found in subjects with $\mathrm{DD}^{13,14}$. It is assumed that the neuroanatomic alterations are responsible for the clinical symptoms. Functional magnetic resonance imaging ( fMRI) studies in subjects with DD have reported alterations also in left-hemispheric activation patterns ${ }^{15}$. Each patient identified with DD merits long-term therapeutic and rehabilitation treatments, which would allow an optimal cognitive development.

Although recognition of frequency; duration of sounds; music pitch, and recognition of environmental sounds of objects and animal calls engage both cerebral hemispheres; the right hemisphere's contribution is predominant in these tasks. Thus, we selected different cerebral right hemispherepreferential stimuli to test CAP in children with DD. We tried to answer the following question; is there any right hemispheric alteration of CAP in right-handed patients with DD when they are compared with healthy control subjects (CS)? Our objectives were to analyze the performance of tests that challenge some central auditory tasks, including recognizing different frequencies patterns, and sound duration; identifying musical patterns, and discriminating between environmental sounds including those produced by different objects and animals, and to relate differences in tests performance with the possible dysfunction of the cerebral right hemisphere in children with DD.

\section{METHOD}

\section{Subjects}

We performed an analytical, prospective, cross-sectional research. The group of children with DD had to fulfill the following inclusion criteria. They should be between 7 and 11 years of age, have adequate visual acuity studied by means of Snellen chart, have adequate hearing acuity studied by means of pure tone audiometry (PTA) and tympanometry, have a normal result in neurological examination, have no psychiatric alterations, have a normal intelligence quotient in the Wechsler intelligence scale for children (WISC), belong to a functional family with adequate scholar opportunities, and have been diagnosed with DD according to Diagnostic and Statistical Manual of Mental Diseases, version IV (DSM-IV, American Psychiatric Association) ${ }^{16}$. Children with DD were studied using a neuropsychological examination for children. The children of group of CS were selected within a healthy and asymptomatic population with regular assistance to school with the same inclusion criteria as that used for children with DD, except absence of DD. They were matched by age, gender, and school grade. Exclusion criteria for subjects from both groups were to have attention deficit-hyperactivity disorder, epilepsy, mental retardation, cerebral palsy, autism, anxiety or depression disorders, stuttering, or other neurological diseases, congenital malformations or metabolic alterations. We studied 20 children with DD and 20 CS. Patients came from the Clinic of Child Neuropsychology of the National Institute of Rehabilitation (INR) in Mexico City. The CS came from the same government public elementary schools of children with DD. In both groups, 14 children were males $(70 \%)$ and six were females (30\%). Distribution by school grade was as follows; one male in first grade; three males and one female in second grade; six males and one female in third grade; three females in fourth grade, and four males and one female in fifth grade. Fifty percent of the children with DD were undergoing speech therapy at the time of the study, the other half were near the onset of rehabilitation treatment at our institution. Clinical, neuropsychological, and CAP examinations were performed for all children with DD and CS on the same day and in the same order. The protocol was approved by the Institutional Ethics and Research Boards and signed informed consent forms were obtained from the parents of the children and are on file.

\section{Handedness assessment}

We utilized the test of Subirana to assess hand, feet, and eye specialization in children with DD and $\mathrm{CS}^{17}$. The test 
is constructed by 14 commands; five commands must be performed by hand, five must be performed with feet, and the remaining four with the eye. The score of the test was constructed by the percentage of correct answers on each side of the body. Body laterality was determined where a subject performed $>70 \%$ of commands of the test with one side of the body.

\section{Language neuropsychological examination}

Verbal language was examined in the following domains. Repetition of syllables, words, pseudo-words, and sentences, and picture identification. We evaluated reading of the following domains; reading of syllables, words, pseudo-words, sentences, and a text; in this case we also measured comprehension and accuracy of reading. Writing domain was evaluated in the following domains; writing of the children's names, dictation of syllables, words, pseudo-words, and sentences. In each domain of the examination, we obtained a score, which was percentile-transformed according to the standardized data for the test for the particular age of children ${ }^{18}$.

\section{Right hemisphere central auditory processing tests}

We utilized several CAP tests to measure items that predominantly activate central auditory right hemisphere functions. In these tests auditory stimuli were presented at a 50 decibel-sound level (dB SL) above the threshold of the subject by means of headphones (TDH-49), stimuli duration was around $7 \mathrm{~s}$, with an inter-stimuli interval of $7 \mathrm{~s}$. Examinations were performed in a sound-proof room fulfilling ANSI S3.6 standards:

- The test of frequency pattern determination(FPD) consisted of mono-aural sequential presentation of six items in each ear. Each item had three sounds of two different tones: 1,122 Hz and $880 \mathrm{~Hz}$. The order of presentation of tones was changed randomly. Thus, children with DD and CS may hear the tones in an order such as high-high-low or low-high-low. Participants must answer aloud the detected tones in the perceived order. We determined the percentage of correct answers.

- The tests of duration pattern recognizing (DPR) included mono-aural presentation of six items in three stimuli of $1,000 \mathrm{~Hz}$ with different lasting times: 250 or $500 \mathrm{~ms}$ in each ear. Stimuli were presented in a random order. Children with DD and CS may hear sounds of large-shortlarge or large-large-short duration. Participants were asked to answer aloud what they heard. We determined the percentage of correct answers.

- The test of music (TM) included 10 items by ear. We mono-aurally administered fragments of popular instrumental known melodies (such as the Mexican national anthem) of around $7 \mathrm{~s}$ of duration, which included guitar, piano, drums, or strings (but without singing). Melodies were modified in tone (low, middle, or high pitch) and presented in pairs. Each stimulus consisted of presentation of some melody at random followed by the same melody modified in pitch frequency. Participants had to identify if the melodies were in different or the same pitch. We determined the percentage of correct answers.

- The test of environmental sound (TES) has two subtests, the first corresponding to sounds produced by different unanimated objects (SPO) and the second, to characteristic sounds produced by the best known animals (SPA), without human voices. In both tests, ten single stimuli were presented in alternate fashion in each ear, and the subjects were asked to identify the nature of sound stimuli. In the SPO tests we included sounds produced by land, water, or air vehicles; phones ringing; water falling; fireguns; glasses broken, and other common sounds. In the SPA test we included sounds from known animals such as dogs, cats, cows, birds, lions, and other animals. The test has been validated in a previous report ${ }^{19}$. We determined the percentage of correct answers.

\section{Statistics}

We measured the mean and standard deviation (SD) of continuous variables and percentages in binomial variables. We utilized the Student's $t$-test to compare means between the groups of children with DD and CS. We measured the force of association among neuropsychological examination and right hemisphere CAP tests by means of the Spearman method. We examined visually each scattergram to elucidate the true significant correlation and avoid statistical artifacts. The $a$-priori alpha value accepted was $p \leq 0.05$. We utilized SPPS software version 17.0.

\section{RESULTS}

\section{General data}

We found that $65 \%$ of children with DD presented family antecedents of language and learning alterations; on the other hand, only $5 \%$ of CS presented family antecedents of language alterations $(p<0.01)$. Forty percent of children with DD had high-risk antecedents for perinatal brain injury; CS presented only $10 \%$ of perinatal antecedents for brain injury $(p<0.01)$. Psychomotor development was normal in 95\% of children with DD as well as CS. Twenty percent of children with DD presented developmental language delay, while all CS presented language development within the normal range $(p<0.05)$.

\section{Psychological and neuropsychological examinations}

All of the children with DD and CS were right-handed and also presented preference for the use of the right eye and the right feet according to the test of cerebral dominance of Subirana ${ }^{17}$. The Wechsler Intelligence Scale for Children (WISC-R) scores were as follows: in children with DD, the 
total IQ average was 97.85 (SD \pm 13.47$)$ while in CS it was 102.1 $(\mathrm{SD} \pm 7.69)$. The mean verbal IQ of the group of children with DD was 96.95 ( $\mathrm{SD} \pm 16.64$ ) while that of $\mathrm{CS}$ was $101.9(\mathrm{SD} \pm 8.58)$. The executive IQ of children with DD was 98.33 (SD \pm 9.86 ), while that of CS was 100.55 (SD \pm 8.24 ). We found significantly lower values in the group of children with DD when compared with that of the CS group for all language domains of the neuropsychological examination, except in the case of word repetition, picture identification, and word reading $(p<0.05)$ (Table 1).

\section{Right hemisphere central auditory processing tests}

In the FPD test we found significantly lower values for each ear stimulation test in children with DD when compared with the values obtained for CS (Table 2). In the DPR test, we found significantly lower averages in children with DD for each ear stimulation test when compared with the values obtained for the CS group (see Table 2). In the TM we found significantly lower performance of children with DD vs CS results (Table 2). In the case of TES, in SPO and SPA subtests, we found, once again, lower performance of children with DD in both subtests when compared with the results from the CS group (Table 2).

We found significant differences between responses from the right and the left ear in SPO and SPA stimulation in children with DD. Differences showed a lower percentage of correct responses during left-ear stimulation in both cases (SPO: $t=3.57, p=0.002$; SPA: $t=2.34, p=0.03$, respectively). We

Table 1. Neuropsychological examination results in children with developmental dyslexia (DD) and control subjects (CS).

\begin{tabular}{|c|c|c|c|c|}
\hline Subtest & $\mathrm{DD}(\mathrm{x} \pm \mathrm{SD})$ & $\mathrm{CS}(\mathrm{x} \pm \mathrm{SD})$ & $\mathrm{t}$ & $p$-value \\
\hline Syllable repetition & $60.80 \pm 14.75$ & $70.20 \pm 6.03$ & -2.63 & $0.01^{*}$ \\
\hline Word repetition & $57.65 \pm 19.72$ & $65.40 \pm 4.92$ & -1.70 & 0.09 \\
\hline Pseudoword repetition & $61.50 \pm 12.63$ & $73.20 \pm 4.39$ & -3.91 & $<0.001^{*}$ \\
\hline Sentence repetition & $60.35 \pm 26.15$ & $84.05 \pm 10.51$ & -3.70 & $0.001^{*}$ \\
\hline Picture identification & $54.75 \pm 19.81$ & $50.00 \pm 1.00$ & 1.07 & 0.29 \\
\hline Command execution & $58.85 \pm 19.02$ & $78.60 \pm 8.73$ & -4.21 & $<0.001^{*}$ \\
\hline Syllable reading & $48.10 \pm 20.24$ & $61.05 \pm 4.76$ & -2.78 & $0.008^{*}$ \\
\hline Word reading & $45.30 \pm 18.97$ & $51.90 \pm 6.16$ & -1.48 & 0.14 \\
\hline Pseudoword reading & $48.00 \pm 19.52$ & $69.40 \pm 10.30$ & -4.33 & $<0.001^{*}$ \\
\hline Sentence reading & $41.40 \pm 22.47$ & $72.30 \pm 6.80$ & -5.88 & $<0.001^{*}$ \\
\hline Sentence comprehension & $46.10 \pm 24.87$ & $76.94 \pm 11.39$ & -4.93 & $<0.001^{*}$ \\
\hline Reading text aloud & $61.10 \pm 28.64$ & $83.30 \pm 12.02$ & -3.62 & $0.001^{*}$ \\
\hline Own name writing & $46.60 \pm 10.46$ & $66.00 \pm 5.33$ & -7.38 & $<0.001^{*}$ \\
\hline Syllable writing & $39.55 \pm 26.71$ & $72.35 \pm 8.76$ & -5.21 & $<0.001^{*}$ \\
\hline Word writing & $50.60 \pm 35.45$ & $84.55 \pm 8.71$ & -4.15 & $<0.001^{*}$ \\
\hline Pseudoword writing & $31.55 \pm 27.63$ & $77.15 \pm 8.31$ & -7.06 & $<0.001^{*}$ \\
\hline Sentence writing & $39.55 \pm 28.56$ & $80.75 \pm 11.16$ & -6.00 & $<0.001^{*}$ \\
\hline
\end{tabular}

x: mean; SD: standard deviation; t: value of Student's t-test; p: value of significance; *statistically significant.

Table 2. Central auditory processing assessment test in children with developmental dyslexia (DD) and control subjects (CS).

\begin{tabular}{|c|c|c|c|c|}
\hline Subtest & $\mathrm{DD}(\mathrm{x} \pm \mathrm{SD})$ & $C S(x \pm S D)$ & $\mathrm{t}$ & $p$-value \\
\hline FPD right ear & $64.47 \pm 21.54$ & $75.80 \pm 17.74$ & -2.23 & $0.03^{*}$ \\
\hline FPD left ear & $66.63 \pm 20.23$ & $79.14 \pm 16.99$ & -2.11 & $0.04^{*}$ \\
\hline DPR right ear & $56.63 \pm 15.63$ & $75.80 \pm 15.73$ & -3.86 & $<0.001^{*}$ \\
\hline DPR left ear & $58.30 \pm 20.58$ & $72.46 \pm 14.58$ & -2.51 & $0.01^{*}$ \\
\hline TM right ear & $82.00 \pm 12.39$ & $96.00 \pm 5.98$ & -4.54 & $<0.001^{\star}$ \\
\hline TM left ear & $87.50 \pm 10.69$ & $97.50 \pm 4.44$ & -3.86 & $<0.001^{\star}$ \\
\hline SPO right ear & $94.00 \pm 8.82$ & $99.50 \pm 2.23$ & -2.70 & $0.01^{*}$ \\
\hline SPO left ear & $87.50 \pm 10.19$ & $96.00 \pm 8.20$ & -2.90 & $0.006^{*}$ \\
\hline SPA right ear & $84.00 \pm 13.13$ & $93.50 \pm 5.87$ & -2.95 & $0.005^{*}$ \\
\hline SPA left ear & $78.00 \pm 13.21$ & $93.50 \pm 6.70$ & -4.67 & $<0.001^{*}$ \\
\hline
\end{tabular}

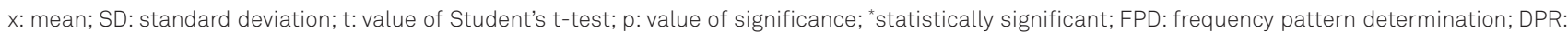
duration pattern recognizing; TM: test of music; SPO: sounds produced by objects; SPA: sounds produced by animals. 
found significant differences between responses from the right and the left ear in FPD, DPR, and SPO stimulation in CS. Differences showed a lower percentage of correct responses during right-ear stimulation in FPD stimulation $(t=-2.17$, $p=0.04$ ); in DPR and SPO stimulation we observed a lower percentage of correct responses during left-ear stimulation (DPR: $t=2.17, p=0.04$; SPO: $t=2.10, p=0.04$, respectively).

\section{Correlations between neuropsychological examination and right hemisphere central auditory processing tests in children with developmental dyslexia}

We found significant correlations among picture identification and left-ear stimulation in the TM test $(r=0.58, p=0.007)$, and between syllable writing and right-ear stimulation for DPR test $(r=0.50, p=0.02)$.

\section{DISCUSSION}

\section{Main findings}

In the present research we found results that support the hypothesis that clinical alterations of spoken and written language in DD are significantly contributed to by cerebral auditory right hemisphere dysfunction. That is, children with DD have more difficulties to process different attributes of sound stimuli using the right hemisphere, such as in recognition of different frequencies and temporal resolution, analysis of musical patterns, and identification of environmental sounds (animated or unanimated).

\section{Explanations and comparison with other studies}

FPD and DPR tests allowed us to examine CAP, interhemispheric communication, and to identify patients with difficulties in supra-segmental information processing ${ }^{20}$. Alterations found in FPD and DPR tests are in line with the hypothesis of the fast and not appropriated cortical processing of the hearing function ${ }^{13,14}$. These theories may help us explain the poor performance of children with DD that we observed when they were faced with auditory discrimination tasks such as those related to FPD and $\mathrm{DPR}^{8,21}$. For language processing, and no-language stimuli decoding, the right hemisphere contributes in a very important way in pitch determination of auditory inputs. In this study we used tonal stimuli, which were modified in frequency (demanding contrast of frequency). Children with DD showed limitations in identifying tonal variations, which show alterations in their auditory perception. However, FPD and DPR tests are in some way unspecific, since in the fashion the test was performed (i.e., with repetition of the subjects heard), it was not possible at all to know whether the successes or errors of subjects primarily involve the right hemisphere, left hemisphere, or inter-hemispheric connections. For a better statement, a future study would require another type of stimulus, such as humming or imitation. Thus, these results must be taken into consideration with caution.

Analysis of musical stimuli alone (without language material as in singing) involves both cerebral hemispheres, although it is traditionally accepted that the right hemisphere is engaged in discrimination of the tone, melody, and the quality of the tone ${ }^{22}$. For this reason, we modified intentionally the tone of the musical stimuli to challenge the music recognizing abilities of children with DD. Again, children with DD presented significantly poor performance in the left-ear stimulation than CS, a fact that suggests a right hemisphere dysfunction. This finding may result from the difficulty of DD children to identify differences between melodic stimuli by the auditory temporal cortex of the right hemisphere. Zatorre and Baum studied influence of pitch determination in music and language; because both share features of a hierarchical structure, complex sound systems, and sensorimotor sequencing demands, it might be natural to assume that pitch processing in speech and music would also depend on the same underlying cognitive and neural mechanisms. In their study, the researchers argue that the processing of pitch information differs significantly for speech and music; specifically, they suggested that there are two pitch-related processing systems, one for more coarse-grained analysis and one for more fine-grained accurate representation, and that the latter is unique to music. They found that music melodic variations of pitch are discontinuous, while in language, the variations are continuous. Isolated alteration of the right hemisphere was related to melodic perception difficulties. Prosodic alterations are also related to the right hemisphere. This study also utilized DFP and $\mathrm{TM}^{23}$. Another explanation of musical processing engages the mirror neuron system (MNS), which is built in a fronto-parietal network architecture. This system is active during action, imitation, and observation. MNS was developed by the need of learning and cognitive-social empathy, overlapping functions of perception, language, and motor cortical areas during music listening, as the transcendent anatomic sites, and those others where emotion lies, such as the limbic system ${ }^{24}$.

At last, when we studied both TES examinations: the SPO and SPA tests; we observed that in the case of both, the results of children with DD were poorer than those of CS. For recognizing unanimated and animated sounds, subjects needed the previous experience of exposure to the sound of the appropriate objects and animals. Children with DD and CS from our sample came from the same city, and from identical public schools in the same geographic metropolitan area. Thus, we assumed that the socio-cultural environment were similar between the groups despite individual differences among subjects. TES studies have revealed in previous investigations that differential cortical auditory analyses are 
needed more than voice stimuli analysis ${ }^{25}$. Results from SPO and SPA tests in our sample showed a left-ear-worse result for the group of children with DD; this fact may also reflect a cerebral right hemisphere alteration in the quality of tone analysis in SPO and SPA tests in our children with DD. This result is supported by another research. Lewis et al. evaluated fMRI 20 right-handed adult subjects using two types of stimuli: animal vocalizations and hand-manipulated tool sounds. The investigators observed differential cortical activation patterns: animal vocalizations activated left and right superior temporal gyri, while hand-manipulated tool sounds activated primarily the left hemisphere, comprising nine areas from frontal, parietal, and temporal lobes, belonging to the cortical MNS engaged with tool work ${ }^{26}$. Results from this research are in good agreement with our data, and suggest that one of the stimuli, TES examination, has major relevance for discovering a cerebral right hemisphere dysfunction in children with DD.

\section{Central auditory processing and hemispheric specialization}

We compared percentages of correct answers between children with DD and CS for right-ear stimulation, for the left-ear stimulation in children with DD and CS, and for CAP and hemispheric specialization measurement. For this purpose, we utilized $t$ of Student- $t$ test for independent samples (Table 1). We observed significant differences ranging between $<0.001$ and 0.04 , the last result in the left-ear stimulation in the FPD subtest. We observed a clear predominance of better performance in the group of CS, especially for the left-ear stimulation with SPO (99\%), and for TM with leftear stimulation (97\%). In contrast, we disclosed a worse performance corresponding to FPD and DPR tests in the group of children with DD, supporting the hypothesis of the right hemisphere dysfunction.
To compare left-right CAP performance in each group, we used $t$ of Student for paired samples. We observed again a lower significant performance for the right-hemisphere in the group of children with DD. The differences were noticeably significant for left-ear stimulation in SPO and SPA in children with DD, while we observed significant differences in left- and right-ear stimulations in the group of CS. These results also support our hypothesis of cerebral right hemisphere dysfunction in CAPD in children with DD.

Finally, correlation analysis confirms some points of our hypothesis. Left-ear stimulation was related to alterations in children with DD, while in CS, alterations were observed for both left- and right-ear stimulation. Thus, data from this research demonstrated that children with DD possess a significant cerebral right hemisphere disadvantage for simple and complex central auditory analysis. However, left hemisphere participation cannot be excluded at all.

\section{Limitations of the study}

Our study has certain limitations. The research had a case and control, cross-sectional design instead of a prospective follow-up design. The number of cases studied is small. Thus, our results show tendencies and not strong conclusions. In future research the number of studied subjects and the period of observation must be increased. In addition, technological devices must be improved in future investigations, i.e., functional neuroimaging and neurophysiological studies will be used in order to obtain better observations.

In conclusion, we found results supporting the idea that simple and complex auditory right hemisphere analysis is altered in children with DD. Thus, cerebral right hemisphere CAP must be considered for examination and rehabilitation of children with DD. Nonetheless, contribution of the left hemisphere in DD genesis cannot be excluded at all. More research is necessary to answer this question.

\section{References}

1. Badzakova-Trajkov G, Häberling IS, Roberts RP, Corballis MC. Cerebral asymmetries: complementary and independent processes. PloSOne. 2010;5:e9682.

2. Arteaga C, Poblano A. Handedness of children determines preferential facial and eye movements related to hemispheric specialization. Arq Neuropsiquiatr 2008;66:488-493.

3. Taylor K, Regard M. Language in the right cerebral hemisphere: contributions from reading studies. News Physiol Sci 2003;18:257-261.

4. Hachinski K, Hachinski V. Music and the brain. Can Med Assoc J 1994:151:293-296

5. Coslett HB, Monsul N. Reading with the right hemisphere: evidence from transcranial magnetic stimulation. Brain Lang 1994;46:198-211.

6. Peñaloza-López Y, Poblano A, García-Pedroza F. Central auditory processing alterations in phonological dyslexia. Horizons in Neuroscience Research. New York, Nova Science Pub. 2013 (in press).

7. Shaywitz SE, Shaywitz BA, Fletcher JM, Escobar MD. Prevalence of reading disability in boys and girls. Results of the Connecticut longitudinal study. JAMA 1990; 264: 998-1002.
8. Tallal P. Auditory temporal perception, phonics, and reading disabilities in children. Brain Lang 1980; 9:182-98.

9. Olivares-García MR, Peñaloza-López YR, García-Pedroza F, PérezRuiz SJ, Uribe-Escamilla R. Identification of auditory laterality by means of a new dichotic digit test in Spanish and body laterality and spatial orientation in children with dyslexia and controls. Rev Neurol 2005;41:198-205.

10. Hoppes E. Lateral asymmetry in subgroups of dyslexic children. Brain Lang 1984;22:221-231.

11. Fisher S, de Fries JC. Developmental dyslexia: genetic dissection of a complex cognitive trait. Nat Rev Neurosci 2002;3:767-780.

12. Ramus F, Rosen S, Dakin S, et al. Theories of developmental dyslexia: insights from a multiple case study of dyslexic adults. Brain 2003;126:841- 865.

13. Livingstone $M$, Rosen $G$, Drislane F, Galaburda A Physiological and anatomical evidence for a magnocellular defect in developmental dyslexia. Proc Natl Acad Sci USA 1991;88:7943-7947. 
14. Galaburda AM, Menard M, Rosen G. Evidence for aberrant auditory anatomy in developmental dyslexia. Proc Natl Acad Sci USA 1994; 91:8010-8013.

15. Heim S, Grande M, Meffert E, et al. Cognitive levels of performance account for hemispheric lateralisation effects in dyslexic and normally reading children. Neuroimage 2010;53:1346-1358.

16. American Psychiatric Association. Diagnostic and Statistical Manual of Mental Disorders, $4^{\text {th }}$ edition (DSM-IV). Washington, American Psychiatric Association. 1994.

17. Subirana A. Handbook of Clinical Neurology. Amsterdam, North Holland. 1969.

18. Matute E, Rosselli M, Ardila A, Ostrosky F. Child Neuropsychology. Mexico City, Manual Moderno. 2007.

19. Romero-Díaz A, Peñaloza-López Y, García-Pedroza F, Pérez SJ, Castro-Camacho W. Central auditory processes evaluated with psychoacoustic tests in normal children. Acta Otorrinolaringol Esp 2011;62:418-424.
20. Jamison H, Watkins K, Bishop D, Matthews P. Hemispheric specialization for processing auditory non-speech stimuli. Cerebral Cortex 2006;16:1266-1275.

21. Musiek F. The frequency pattern test: a guide. Hear J 2006;55:58-66.

22. Brancucci A, Babiloni C, Rossini P, Romani G. Right hemisphere specialization for intensity discrimination of musical and speech sounds. Neuropsychologia 2005;43:1916-1923.

23. Zatorre RJ, Baum SR. Musical melody and speech intonation: singing a different tune. PloS Biol 2012;10:e1001372.

24. Minar-Szakacs I, Overy K. Music and mirror neurons: from motion to 'e'motion. Soc Cogn Affect Neurosci 2006;1:235-241.

25. Suied C, Viaud-Delmon I. Auditory-visual object recognition time suggest specific processing for animal sounds. PLoS-One 2009;4:e5256.

26. Lewis JW, Brefczynski JA, Phinney RE, Janik JJ, de Yoe EA. Distinct cortical pathways for processing tool versus animal sounds. J Neurosci 2005;25:5148-5158. 